\title{
INCREASE OF POSITRONS BY A HIGH-INTENSITY TWO-BUNCH ACCELERATION SCHEME AT THE KEKB LINAC
}

\author{
Satoshi Ohsawa, Atsushi Enomoto, Eiji Kikutani, Kazuro Furukawa, Naoko Iida, Mitsuo Ikeda, \\ Norihiko Kamikubota, Takuya Kamitani, Hitoshi Kobayashi, Haruyo Koiso, Toshihiro Matsumoto, \\ Yujiro Ogawa, Yukiyoshi Ohnishi, Katsunobu Oide, and Tsuyoshi Suwada, KEK, Tsukuba, Japan
}

\begin{abstract}
As the accumulation current of positrons increases in the KEKB ring, the injection time is becoming longer. It will thus be one of the most important issues affecting the accumulation of the integrated luminosity. As one of the steps, we introduced a high-intensity two-bunch acceleration scheme at the KEKB linac to intensify positrons by means of doubling the primary electrons. We recently obtained test results of $0.54 \mathrm{nC}$ for the first bunch and $0.49 \mathrm{nC}$ for the second bunch at the linac end. This scheme increased the positron intensity by nearly $65 \%$. Since the linac frequency is not a harmonic number of the LER frequency, the best time interval between two bunches is $96.29 \mathrm{~ns}$, corresponding to 49 LER-buckets. Even with this limitation, it is undoubtedly a very useful scheme for increasing the positron injection rate. The beam test results are described.
\end{abstract}

\section{INTRODUCTION}

From the beginning of the KEKB project, we have only been accelerating S-band single bunch beams at the KEKB linac for injection into the KEKB rings. The reasons why we have used the single bunches are as following: 1) Since wake field effects produced by proceeding bunches would be strong in the case of highintensity multi bunches, it is easy to avoid the effects for stable operation. 2) Since the bunch separation is 350ps in the linac, the s-band multi bunches do not satisfy the KEKB requirement that the beams should be injected within a time range of $\pm 30 \mathrm{ps}$ against the ring RF buckets.

As the LER accumulation current is increasing, the subject of intensifying positrons is becoming more important. We have thus introduced a two-bunch scheme in order to double the total charge of the primary electron beams for increasing positrons. In order to satisfy the second condition, the bunch separation was determined so as to be $96.29 \mathrm{~ns}$, which is the period of the common frequency $(10.3 \mathrm{MHz})$ of both the linac and the KEKB rings. The separation is conveniently sufficiently longer than the RF filling times of the prebuncher and the bunching section of the buncher, which are 8 ns and 48 ns, respectively. Therefore, it is expected that in the bunching process the second bunch would not be affected by the wake fields produced by the first bunch in the prebuncher and the buncher because the RF waves are replaced before the second bunch arrives at the devices, which are both of travelling wave type.

An energy difference occurs between two bunches due to the time-varying SLED pulse and beam loading. Energy compensation can be carried out by means of optimising the SLED pulse timing against the two bunches in order to equalize the two-bunch energies [1].

We experimentally examined the feasibility of the twobunch scheme. The results are very satisfactory, which shows that the two-bunch scheme is promising to improve the positron injection rate.

\section{TWO-BUNCH BEAM GENERATION}

\subsection{Double-Pulse Production for the Gun}

First of all, a new grid-pulser system for the electron gun has been developed that independently produces two pulses to execute the two-bunch scheme [2]. The system mainly consists of a pair of same grid pulsers with timedelay units and four combiners that were newly developed to combine high-voltage, short pulses of $1 \mathrm{kV}$ and $1 \mathrm{~ns}$. It can produce single pulses for single-bunch operation as well as double pulses for two-bunch operation. The bunch-selector system gives inhibit signals for the time-delay modules to choose any pulses. It will automatically determine the operation mode; namely, the single-bunch or two-bunch modes will be selected rapidly at injection into the LER.

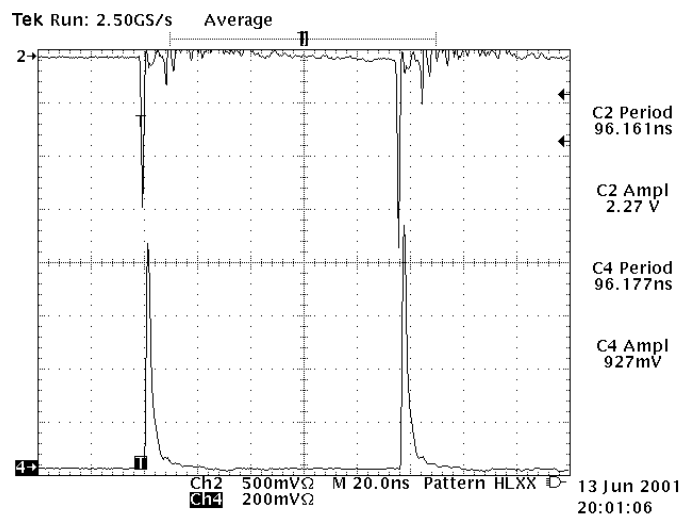

Figure 1: Typical example of double grid pulses (upper), and the electron beam from the gun corresponding to the grid pulses (lower). The pulse 
heights and timings were changed as free parameters, and determined so that the two-bunch charges and orbits coincide with each other. Therefore, the peak intensities are not the same, and the time interval is different from $96.29 \mathrm{~ns}$, which is the period of the common frequency.

The time-delay module has two functions: 1) it produces stabilized trigger signals synchronized with an RF wave of $571.2 \mathrm{MHz}$, and 2) it changes the trigger time delay with steps of $1.75 \mathrm{~ns}$ over a wide range of more than $100 \mathrm{~ms}$. Each delay module roughly determines the pulse timing independently. Any trigger pulses can be inhibited at the delay modules with the signals from the pulse selector system.

The grid pulser has the following two functions: 1) a fine timing control that continuously changes within a range of $3 \mathrm{~ns}$, and 2) a peak voltage control for output pulses. These values are controlled with DC signals $(0 \sim 10 \mathrm{~V})$ from a PLC. For stable operation, the pulse timings are, externally controlled with a feedback system that adjust the fine timings. Four output ports of the coaxial type are used in order to make the source impedance low.

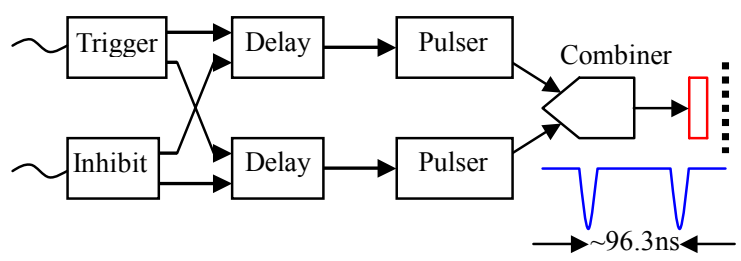

Figure 2: New grid pulser system for the two-bunch acceleration scheme.

Pulser No.1

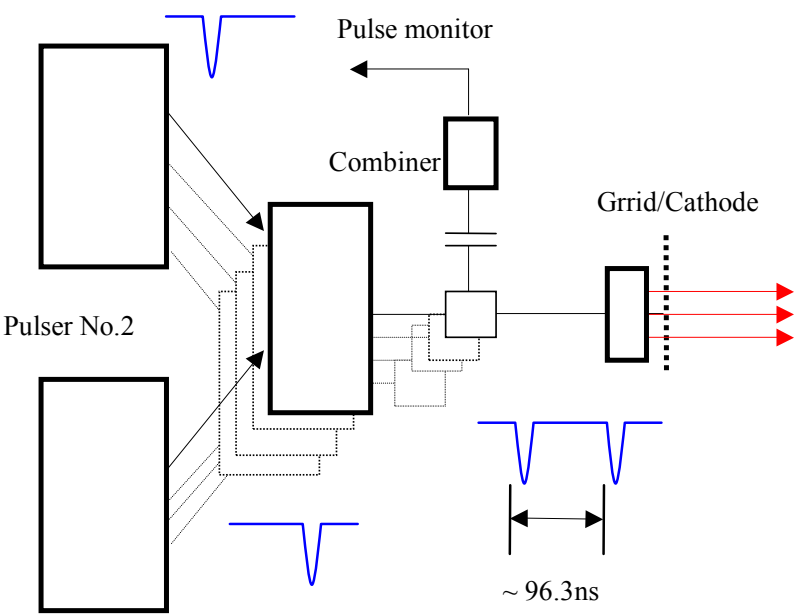

Figure 3: Combiners make double pulses from pairs of independent pulses. The pulse heights and timings are free parameters.

A pulse combiner was introduced to make double the pulses from pairs of single pulses that are produced by independent pulsers, and simultaneously to prevent any interference between the pulsers in order not to damage each other. Since a grid pulser has four output ports, four combiners are used.

The monitor signals of the pulses are transported through an optical fiber to a digital oscilloscope on the ground level. The pulse timings are measured relatively with respect to an RF wave of $571 \mathrm{MHz}$, and are used in the digital feedback system to stabilize the pulse timings.

Double electron pulses from the gun are compressed into two bunches in the bunching section of the preinjector by two sub-harmonic bunchers (SHBs) operated at frequencies of $114.24 \mathrm{MHz}$ and $571.2 \mathrm{MHz}$, and by a prebuncher and a buncher operated at $2856 \mathrm{MHz}$.

\subsection{Two-bunch Beam Generation}

In the SHBs, which are single cavities of resonance with high-quality factors, the wake fields induced in the cavities do not damp effectively during the bunch time interval of $96 \mathrm{~ns}$. The amplitudes of the wake fields due to a beam of $13 \mathrm{nC} /$ pulse are, however, still rather smaller than those of the fundamental RF fields, as is shown in Fig. 5. Therefore, the SHBs still retain the bunching function, although the induced fields deform the bunch shapes.

Although beam acceleration was successful, the bunch profiles observed with a streak camera had rather large tails. Further investigations and fine-tuning are required in order to improve the shapes, if necessary, with more sophisticated beam diagnostic tools. We could expect further increases of positrons.

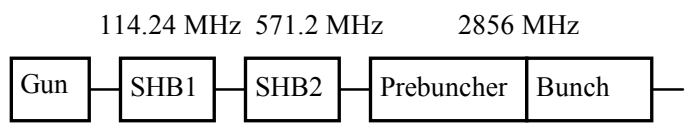

Figure 4: Main components of the bunching section.

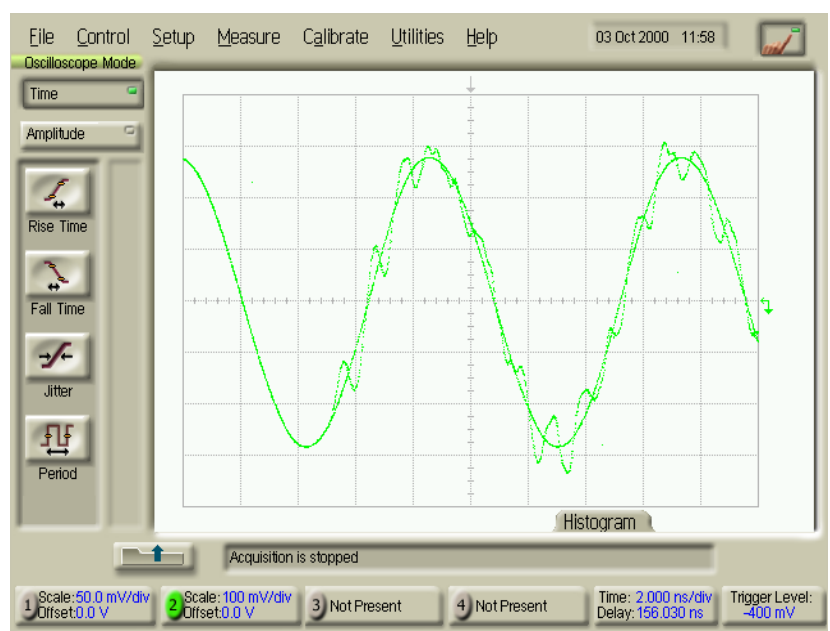

Figure 5: Typical RF wave in the first SHB (114.24 $\mathrm{MHz}$ ). The wake fields induced by the $13-\mathrm{nC}$ beam are superposed on the fundamental RF wave. Since the beam repetition rate is different from the RF, deformation due to the wake fields is clearly seen. 

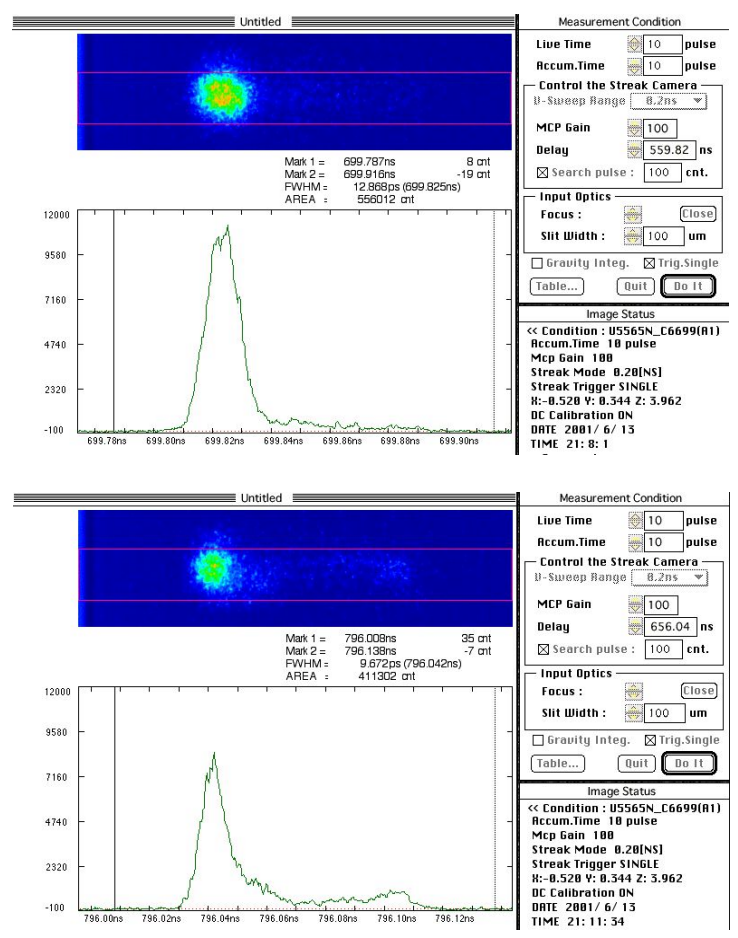

Figure 6: Bunch shapes of the 1st bunch (upper) and the 2nd bunch (lower), which were measured with a streak camera located at the end of the pre-injector.

\section{TWO-BUNCH ACCELERATION RESULTS}

We have achieved the successful acceleration of highintensity, two bunches with charges of $7.8 \mathrm{nC}$ and $8.0 \mathrm{nC}$ to a positron target with a small loss, which produced positron bunches with charges of $0.54 \mathrm{nC}$ and $0.49 \mathrm{nC}$ at the end of the linac, respectively. The sum of the positrons reached to $165 \%$ of the single bunch charge that actually injected into the ring during the two-bunch acceleration test.

Figure 7 shows a typical example of two bunches. The horizontal and vertical orbit displacements ( $\mathrm{dx}$ and $\mathrm{dy}$ ) and the bunch charges are shown. The blue points show the values of the first bunch, and the green ones show the second bunch. The data of the two bunches are shown on the same corresponding frames.

Table 1: Beam charges and the enhancement factors against a single bunch during normal operation.

\begin{tabular}{|l|l|l|l|}
\hline $\begin{array}{l}\text { Position of } \\
\text { measurement }\end{array}$ & $\begin{array}{l}1^{\text {st }} \text { bunch } \\
\text { charge }\end{array}$ & $\begin{array}{l}2^{\text {nd }} \text { bunch } \\
\text { charge }\end{array}$ & $\begin{array}{l}\text { Enhancement } \\
\text { factor }\end{array}$ \\
\hline Electron & & & \\
\hline$@$ buncher & $9.54 \mathrm{nC}$ & $9.59 \mathrm{nC}$ & $179 \%$ \\
\hline$@$ target & $7.79 \mathrm{nC}$ & $8.02 \mathrm{nC}$ & $190 \%$ \\
\hline Positron & & & \\
\hline$@$ target & $0.88 \mathrm{nC}$ & $0.90 \mathrm{nC}$ & $170 \%$ \\
\hline$@$ linac end & $0.539 \mathrm{nC}$ & $0.486 \mathrm{nC}$ & $165 \%$ \\
\hline
\end{tabular}

In two-bunch acceleration, first of all, almost all of the parameters were fixed for the first bunch to accelerate it well. We then proceeded to the next step to coincide the second-bunch energy, orbit and charge to the first ones along the linac. We matched the second bunch by changing the gun-beam intensity and timing that were independent parameters in the region following the buncher. We then optimised the pulse timings of the SLED RF waves against the two bunches in order to coincide the bunch energies. This procedure was also useful to reduce an orbit difference between the two bunches.

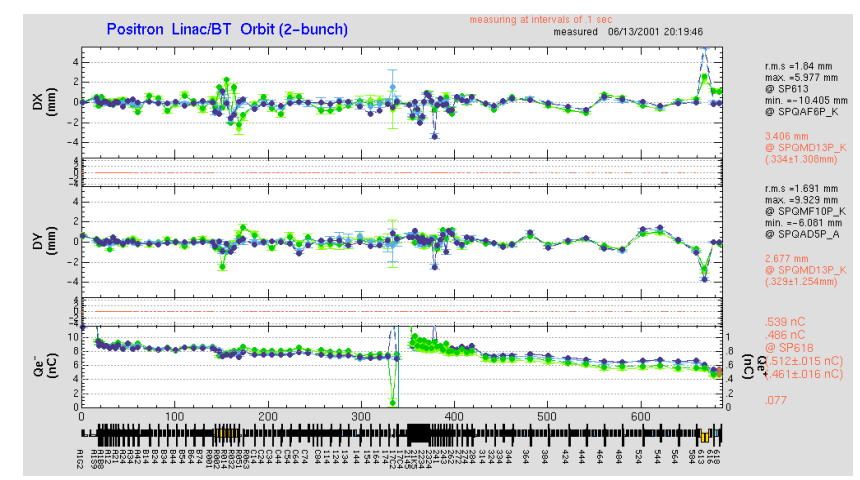

Figure 7: 2-bunch beam orbits and charges along the whole linac. A positron target is located at the center. The blue/green points show values of the $1 \mathrm{st} / 2 \mathrm{nd}$ bunch.

\section{CONCLUSIONS}

We have succeeded to increase positrons by a factor of $65 \%$ by means of introducing a high-intensity, two-bunch acceleration scheme at the KEKB linac. This method opened a way to the short injection of positrons. Two bunches of positron beams with charges of $0.54 \mathrm{nC}$ and $0.49 \mathrm{nC}$ were successfully accelerated to the end of the linac. Their behaviour was surprisingly similar along the linac despite undesirable bunch profiles with rather large tails. When the bunch shapes were improved with further careful fine-tuning, nearly twice as many positrons could be expected.

\section{ACKNOWLEGIMENTS}

We would like to express our special appreciation to Prof. Motohiro Kihara for his encouragement and support. We also wish to express our thanks to the members of the KEKB linac and machine operators for their continuous efforts to maintain the linac in good condition, which is important for this improvement.

\section{REFERENCES}

[1] Y. Ogawa et al., "High-Intensity, Two-Beam Acceleration for Doubling Positron Production at the KEKB Linac, " HEAC'01, Tsukuba, March 2001.

[2] S. Ohsawa et al., "Two-Bunch Beam Generation at KEKB Linac," HEAC'01, Tsukuba, March 2001. 